\title{
Contrôle total
}

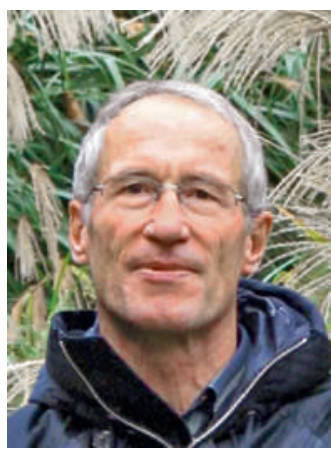

Erhard Taverna
Evaluer les choix technologiques ressemble à la fameuse course du lièvre et du hérisson. A la fin du conte de Grimm, le lièvre épuisé s'écroule et meurt un sort jusqu'à présent épargné à TA-SWISS*, dont une étude récente relève certains aspects problématiques des technologies de localisation et énonce des recommandations envers les consommateurs et les politiques.

Mais les nouveaux coureurs ont depuis longtemps déjà distancé le lièvre. Intitulées self tracking, body hacking ou self quantifying, leurs activités se sont étendues depuis 2007 à tous les continents: des hommes et des femmes collectent leurs données biométriques et les transmettent à leurs amis ou, à fins d'évaluation, à une organisation centralisée telle que www.tictrac.com dont le siège est au RoyaumeUni. Le sport d'élite et la télémédecine ont pris de l'avance. Actuellement, des entreprises comme Philips, Vodafone ou Intel parrainent généreusement les rencontres des fans de technologie dans plus de 70 villes et 26 pays.

Les outils de mesure sont de plus en plus petits, efficaces et bon marché. Des applis comme la digifit d'Apple, iCardio, iRunner, iBiker, iSpinner ou iPower mesurent pulsations cardiaques, pas, rythme ou consommation de calories et affichent des diagrammes à l'écran. Le bandeau de Zeo enregistre le sommeil REM en lien avec la nicotine, l'alcool, l'alimentation, le magnésium et l'activité physique et transfère ces données vers le site Internet du fournisseur. Le Spiroscout d'Asthmapolis analyse des données GPS pour éviter les lieux allergisants, le Boozerlyzer, appli pour smartphones Android, mesure temps de réaction, mémoire et état général après une consommation d'alcool. Nintendo a développé des jeux de compétition qui évaluent et récompensent des activités quotidiennes selon un système de points. Les MoodPanda, MoodScope, Fitbit, Momento ou DailyMile fournissent sans fil d'énormes quantités de données en association avec des sites Web, Facebook, Twitter, Flickr ou des services de conseil, informations, publicités et de la musique issue d'Internet.

Des dizaines de milliers de gens échangent leurs résultats, utilisant des forums comme PatientsLikeMe ou CureTogether pour partager leurs expériences concernant médicaments et thérapies. Des clips, bracelets, patchs, montres et capteurs de mouvement mesurent d'autres fonctions corporelles ou peuvent être fixés sur divers objets - brosse à dent, collier de chien, verres, etc. - afin de consigner autant d'habitudes que possible. D'autres projets sont sur les rails, tels l'affective computing qui reconnait automatiquement le stress ou l'emotional-social intelligence toolkit, censé aider notamment les autistes.

Cartes SIM, GPS, lecteurs de cartes de crédit, capteurs médicaux et caméras vidéo permettent dès aujourd'hui de nous suivre intégralement à la trace. Mais qui se reconnait dans le miroir de ses propres données ne tient pas compte de ces réserves. Les adeptes de l'automesure veulent utiliser la technique pour optimiser leur santé et leurs performances. Ils se considèrent comme des pionniers de la prévention, l'analyse des données devant améliorer notre style de vie et permettre de faire des économies de santé publique. Si le narcissisme, le besoin de communiquer, le goût du jeu et la consommation sont peutêtre les mobiles d'une génération habituée aux ordinateurs, exploitant sans réflexion tous les moyens numériques, celle-ci n'en est pas moins une avantgarde. Mais pour qui? Pour quoi?

La demande des mordus de technique stimule l'industrie électronique, dont les innovations excitent à leur tour la demande du marché. «Je compte, donc je suis»: telle est la devise. Sans calculette, point de salut. Outre le manque de protection des données, on peut ici critiquer l'automesure comme signe d'un manque de confiance en soi, la métamorphose illusoire d'une vie en colonnes de chiffres, le potentiel totalitaire - si le hobby de quelques cinglés se muait en obligation sociale, etc. Les déclarations d'intervenants sur des blogs soulignent le contrôle par les pairs et l'occasion unique de générer de nouveaux savoirs. Ils célèbrent dans le mouvement «Quantified Self» la réconciliation de la science et de la technologie et comparent les rituels de la communauté à une prière. Il faudrait que la politique prenne conscience de la blogosphère comme d'une démocratie directe innovante.

L'économie l'a fait depuis longtemps. Peut-être est-il possible de séparer le bon grain de l'ivraie, les effets placebo des résultats exploitables? D'extraire de ces données des enseignements constituant un progrès pour la médecine? De plus en plus d'hôpitaux, de caisses-maladie et d'assurances s'intéressent aux rencontres annuelles (Amsterdam en 2011, Berlin cette année). Il est certes possible que cette tendance génère quelques avantages pour tous, y compris pour ceux qui n'ont pas envie de dire au monde entier combien de fois par jour ils vont aux toilettes ou promènent le chien. Mais le prix à payer est trop élevé.

Erhard Taverna 